\title{
Thermal tuning of a silicon photonic crystal cavity infilled with an elastomer
}

\author{
A. Koray Erdamar*a , Matheus M. van Leest ${ }^{\mathrm{a}}$, Stephen J. Picken ${ }^{\mathrm{b}}$, J. Caro ${ }^{\mathrm{a}}$ \\ ${ }^{a}$ Kavli Institute of Nanoscience, Department of Quantum Nanoscience, Delft University of \\ Technology, Lorentzweg 1, 2628 CJ Delft, The Netherlands; \\ ${ }^{b}$ Department of Chemical Engineering, Section NanoStructured Materials, Delft University of \\ Technology, Julianalaan 136, 2628 BL Delft, The Netherlands
}

\begin{abstract}
Thermal tuning of the transmission of an elastomer infilled photonic crystal cavity is studied. An elastomer has a thermal expansion-induced negative thermo-optic coefficient that leads to a strong decrease of the refractive index upon heating. This property makes elastomer highly suitable for thermal tuning of the transmission of a cavity, which is demonstrated by global infilling of a hole-type silicon photonic crystal slab and global thermal tuning. In the temperature range 20-60 ${ }^{0} \mathrm{C}$ the cavity peak shows a pronounced elastomer-induced blue shift of $2.7 \mathrm{~nm}$, which amply overcompensates the red shift arising from the thermo-optic property of the silicon. These results qualify the elastomer for tuning by local optical heating.
\end{abstract}

Keywords: Photonic crystal, elastomer, thermo-optic coefficient, cavity, tuning.

\section{INTRODUCTION}

Photonic crystals $(\mathrm{PhCs})$ are artificial dielectric materials with a periodic modulation of the dielectric constant. The most striking property of a $\mathrm{PhC}$ is the photonic band gap, i.e. a wavelength range where the $\mathrm{PhC}$ is opaque. The bandgap and the possibility to engineer its position in wavelength enable the control of the propagation of light [1]. Using these properties, novel devices have been proposed and demonstrated. In a PhC light can be confined in cavities, of which the transmission can be changed via a tunable refractive index. These properties of a cavity are highly attractive for applications such as optical switches [2], add-drop filters [3] and low-threshold lasers [4]. These examples indicate that active tuning of $\mathrm{PhC}$ devices is indispensible for applicable functionalities. The refractive index can be tuned with optical [5], mechanical [6] and electrical methods [7]. Another method is infilling the air hole(s) of a cavity in a PhC with a liquid crystal. The cavity transmission can then be tuned by driving the liquid crystal through the phase transition [8].

\subsection{Cavity tuning with elastomer}

In this work, we apply an elastomer as infill material to tune the transmission of a PhC cavity. Elastomers have weak bonding forces between the polymer chains, which leads to strong a thermal expansion and consequently a high negative thermo-optic coefficient (TOC) $d n / d T$. For infilling and transmission tuning we use Kraton SEBS G 1657 [9] (further just called "elastomer"), which is a highly stable and durable elastomer for infilling. The index of the elastomer is temperature dependent $n_{\text {elast }}(T)=1.478-3.32 \times 10^{-4}\left(T-T_{0}\right)$ in the range of 293-323 K $\left(T_{0}=293 \mathrm{~K}\right)$, as deduced from refractometry measurements at $1550 \mathrm{~nm}$.

*a.k.erdamar@tudelft.nl; phone: +31 152787521.

Active Photonic Materials IV, edited by Ganapathi S. Subramania, Stavroula Foteinopoulou, Proc. of SPIE Vol. 8095, 80951J · @ 2011 SPIE · CCC code: 0277-786X/11/\$18 · doi: 10.1117/12.893940 
For the tuning to be virtually lossless, the absorption of the elastomer should be weak in the wavelength range of the measurements (around $1550 \mathrm{~nm}$ ). That this is indeed the case can be seen in Figure 1, which shows the absorbance as a function of wavelength. The data in this figure result from measurement of the light intensity transmitted through a 150 $\mu \mathrm{m}$ thick elastomer layer deposited on a quartz substrate. The absorbance thus includes absorption and scattering by the elastomer and reflection from interfaces, so that a deduced attenuation coefficient from these data gives an upper bound of the absorption coefficient. As can be seen, the absorbance is rather low and flat between 1200 and $1650 \mathrm{~nm}$, while above this range it shows peaks at 1720 and $2300 \mathrm{~nm}$ due to vibrational modes, the latter one being very pronounced. At $1550 \mathrm{~nm}$ the absorbance amounts to 0.0026 , which corresponds to a maximum possible absorption coefficient of 0.405 $\mathrm{cm}^{-1}$. Thus, on the scale of the elastomer thicknesses relevant in this work absorption is negligible. The peak at $2300 \mathrm{~nm}$ may be strong enough to induce heating effects by absorption, which is promising for tuning applications in the midinfrared.

With the experiments, global infilling of a hole-type silicon PhC slab with the elastomer and global thermal tuning of its refractive index is demonstrated. In particular, we study the tuning properties of the transmission of a cavity in the elastomer infilled $\mathrm{PhC}$ as a function of temperature. Our approach goes beyond the operation regime of athermal waveguides [10], which are clad by a polymer with a negative TOC of the silicon waveguide to make the properties temperature insensitive.

The final tuning goal on the basis of the elastomer is local infilling and local optical heating of the elastomeric infill of a cavity, resulting in an index change and a shift of the resonance wavelength. The absorption of the elastomer being low around $1550 \mathrm{~nm}$, local heating should then be accomplished by doping the elastomer with dye molecules or quantum dots, provided that these materials after light absorption mainly decay non-radiatively, to ensure efficient conversion of locally deposited optical energy into heat [11]. With the global infilling and global tuning experiments presented here we qualify the elastomer for tuning by local heating.

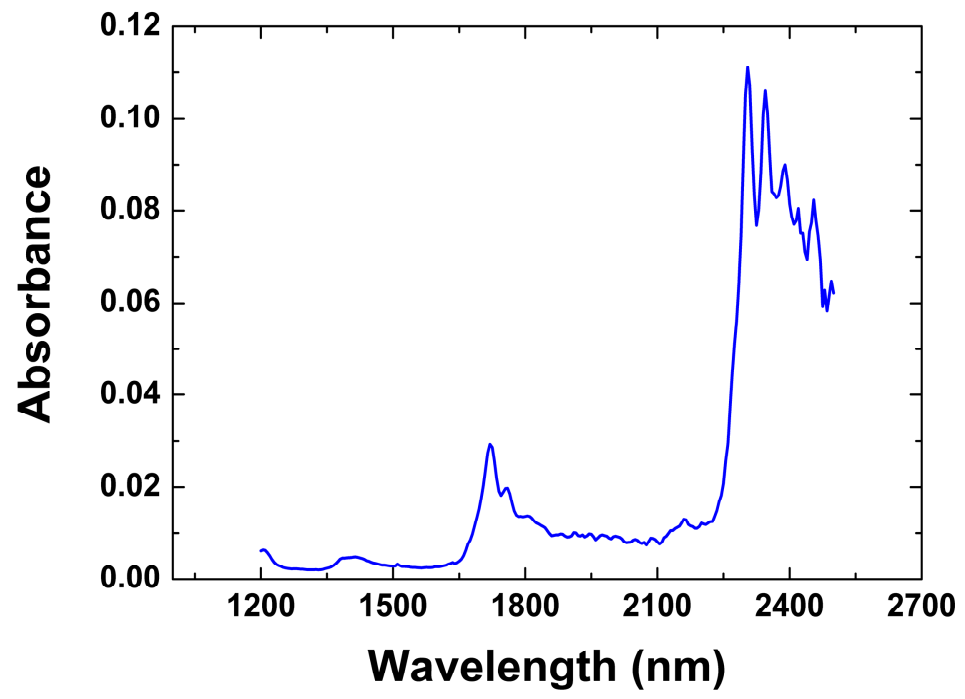

Figure 1. Absorbance of the bulk elastomer. Absorbance includes absorption, reflection and scattering. The thickness of the bulk elastomer is $\approx 150 \mu \mathrm{m}$.

\section{DESIGN AND DEVICE FABRICATION}

Figure 2(a) shows a scanning electron microscope (SEM) image of the design of the cavity including the superimposed mode profile. The cavity, centered between the waveguides, is the modified point-defect cavity of Loncar et. al. [12]. The PhCs have a triangular lattice with nine rows of holes between the waveguides and are oriented for light propagation in the $\Gamma \mathrm{M}$ direction. The designed ratio $\mathrm{r} / \mathrm{a}$ of the hole radius to the lattice constant is 0.3 . The center modified hole has $\mathrm{r} / \mathrm{a}$ ratio of 0.2 . The four holes closest to central defect hole are reshaped into half ellipses with the ellipticity 0.2 . The 
cavity is very sensitive to an index change of the infill, since an appreciable part of the mode energy is localized in the holes. The lattice constant is litho-tuned in the range $490-510 \mathrm{~nm}$ across the PhCs on a chip.

The PhCs are fabricated in silicon-on-insulator (SOI) material, using electron-beam lithography and dry etching. The device layer of the SOI is $220 \mathrm{~nm}$ thick, while the buried oxide is $2 \mu \mathrm{m}$ thick. The lithographic pattern with PhCs and 2.5 $\mu \mathrm{m}$ wide waveguides for in-and outcoupling of near-infrared light is written in a $120 \mathrm{~nm}$ thick ZEP 520 resist with a Leica $5000+$ e-beam system. After resist development the pattern is transferred to the silicon layer using inductively coupled plasma etching in an $\mathrm{SF}_{6}$-based plasma at a substrate temperature of $-120{ }^{\circ} \mathrm{C}$. The final step is creating the membrane PhCs by selectively etching $350 \mathrm{~nm}$ of the buried oxide in buffered HF which is seen in Fig. 2(b).

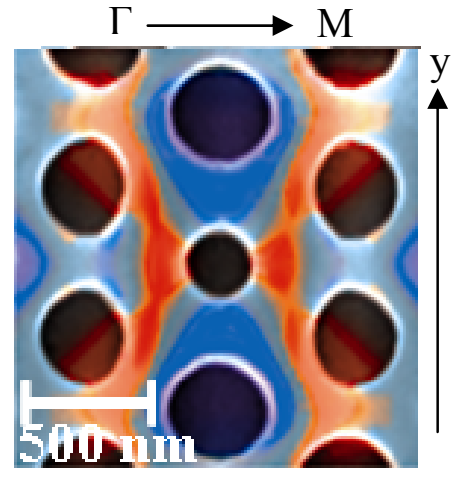

(a)

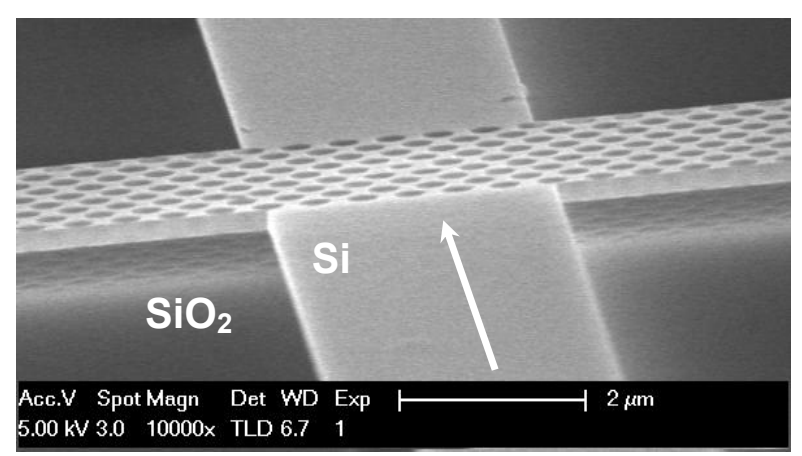

(b)

Figure 2. (a) SEM image of the cavity including the mode profile superimposed ( $\mathrm{E}_{\mathrm{y}}$-field). The r/a ratio of the PhC is 0.32 , while the modified central hole has $\mathrm{r} / \mathrm{a}=0.22$ and the half ellipses have ellipticity of 0.2 . (b) Membrane $\mathrm{PhC}$ device. The arrow shows the propagation direction of the light through the waveguide.

After optical measurements of the empty crystal (i.e. holes not infilled), the chip is cleaned in isoproponol and dried with nitrogen gas, as a preparation for infilling. Infilling is performed with by applying a droplet of $2 \%$ solution by weight of elastomer in cyclohexane. Owing to its low viscosity and good wetting properties, the solution quickly spreads over the $\mathrm{PhCs}$, flows through the holes and fills the space below the membrane, resulting in infilled PhCs with elastomer upper and lower cladding. These features can be seen in Fig. 3, which is a SEM image of an infilled device. The measurements are performed after a certain time, to make sure that the solvent has completely diffused out of the elastomer, as indicated by the stabilization of the spectral features.

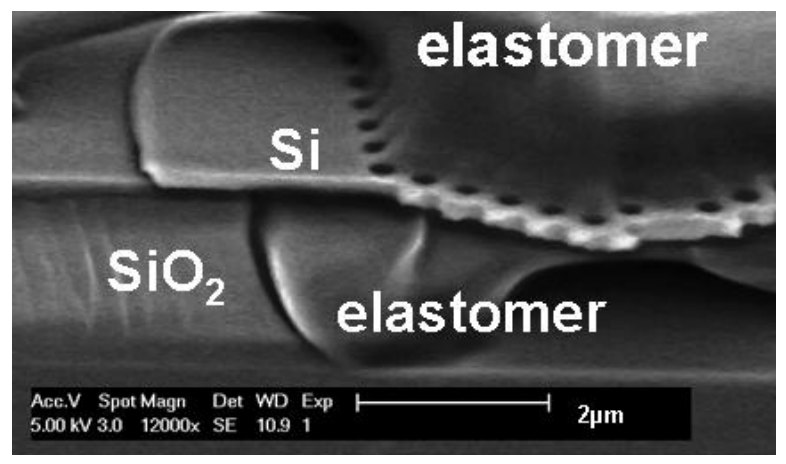

Figure 3. SEM image of a cleaved elastomer infilled PhC. 


\section{TRANSMISSION MEASUREMENTS}

\subsection{Optical measurement set-up}

Optical transmission measurements are performed with the end-fire-technique using, a supercontinuum white light source (Fianium SC 400) in combination with a prism based spectral selection unit [13], giving a wavelength range 1300-1700 nm for the measurements. For excitation of transverse electric PhC modes, the polarization is selected inplane with a polarizer. The light transmitted through the $\mathrm{PhC}$ is measured with an optical spectrum analyzer (ANDOAQ6315A). A temperature controller (Conductus LTC-10) is used to regulate the temperature of the chip, which is working with a heating resistor and a Pt100 temperature sensor mounted on the sample holder. In the temperature range $20-60{ }^{\circ} \mathrm{C}$ the resulting accuracy of the sample-holder temperature is better than $0.2{ }^{\circ} \mathrm{C}$.

\subsection{Infill effect}

Fig. 4 shows the room-temperature transmission spectrum of one of the $\mathrm{PhCs}$, in a range covering the air band edge and part of the bandgap, both for the empty and infilled situation. For the empty case the steep side at $1365 \mathrm{~nm}$ is the air band edge, while the strong peak at $1436 \mathrm{~nm}$ is the cavity resonance. These experimental positions show good aggrement with the positions from the transmission spectrum we calculated with the finite-difference-time-domain (FDTD) simulator CrystalWave [14], as indicated with the blue vertical stripes in the upper part of the Fig. 4. After infilling, in spite of the decreased index contrast $\left(n_{\text {elast }}=1.478\right.$ at $\left.20{ }^{\circ} \mathrm{C}\right)$ we still clearly observe the main features of the spectrum, which exhibits a pronounced red shift, as expected. The infill effect on the air band edge and on the cavity resonance is clearly observed. The air band edge starts from $1500 \mathrm{~nm}$ and the cavity resonance is at $1570 \mathrm{~nm}$, the shift in either case being about $135 \mathrm{~nm}$. The FDTD simulations after infilling predict a stronger shift, as indicated by the red vertical stripes in Fig. 4. This difference will be discussed later in the text.

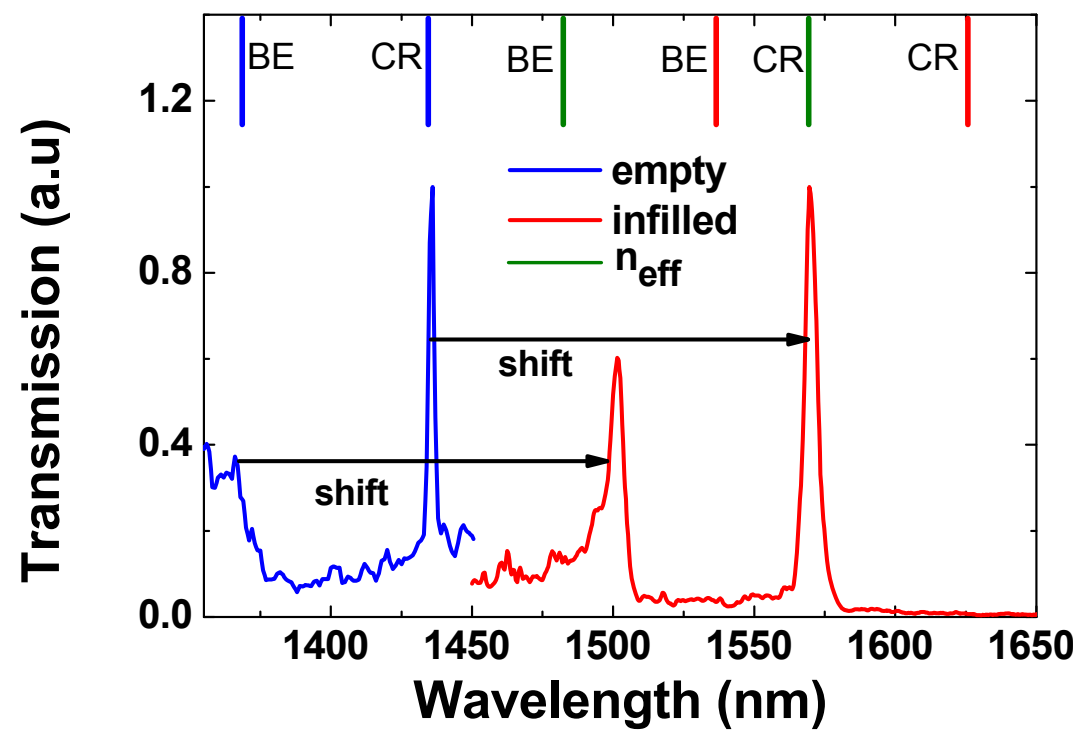

Figure 4. Transmission spectrum of empty (blue) and infilled (red) $\mathrm{PhC}$, showing the air-band edge and the cavity resonance, the spectra normalized to unity for the cavity peak. The blue and red stripes denote simulated positions of the band edge (BE) and the cavity resonance (CR). The green stripes denote these positions for an index $n_{\text {eff }}$. 


\section{GLOBAL THERMAL TUNING OF THE CAVITY RESONANCE}

In Fig. 5(a) we zoom in on the cavity peak, showing it in detail for nine temperatures. In addition to the temperature dependence of the peak after infilling shown in right panel of Fig. 5(a), we also present the data for the empty case in the left panel of Fig. 5(a), for reference. In the right panel, we observe the elastomer induced thermal tuning. With increasing temperature from $20{ }^{\circ} \mathrm{C}$ to $60{ }^{\circ} \mathrm{C}$ the cavity resonance peak shows a steady blue shift. After the temperature dependent transmission measurement for silicon cavity resonance shows a red shift due to having a positive TOC [15]. From comparison of empty and infilled $\mathrm{PhC}$ measurements, we conclude that the negative TOC of the elastomer overcompensates the positive TOC of the silicon. In addition, we observe the broadening of cavity resonance that shows the reduction of the quality factor which will be discussed below.

In Fig. 5 (b), peak shifts as a function of temperature for empty and infilled case are shown, which are obtained from the measurements and FDTD simulations, in which temperature dependent refractive index of silicon $n_{s i}(T)=3.48+1.85 \times 10^{-4}\left(T-T_{0}\right)$ and aforementioned $n_{\text {elast }}(T)$ are used. From the linear fits to data points for the empty case the total shift amounts are $2.1 \mathrm{~nm}$ and $2.4 \mathrm{~nm}$, for the measurements and the simulations, respectively. This is considered as a good agreement. However, after infilling, the experimental and simulation data show an inconsistency. Over the total temperature range, the total amount of shift is $-2.7 \mathrm{~nm}$ for measurements, while it is $-4.4 \mathrm{~nm}$ for the simulations.
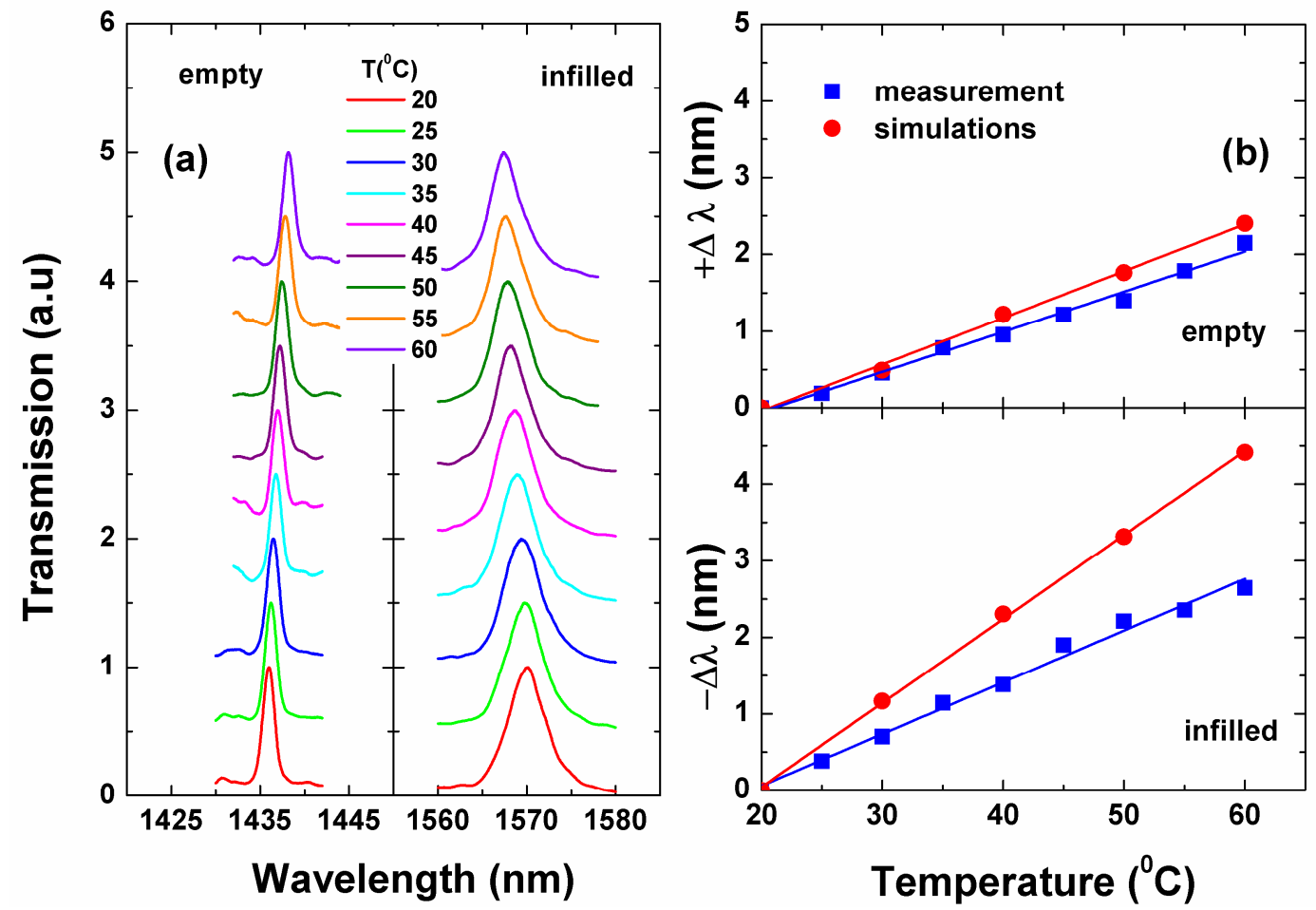

Figure 5. (a) Cavity resonance as a function of temperature for the empty and infilled PhC. The cavity resonance is normalized to unity and offset for clarity. (b) Temperature dependence of the measured and simulated shift of the cavity resonance, for the empty and infilled PhC. The lines are fits to data points. 
The thermal tuning behavior can be understood from the perturbation expression for the relative shift of the resonance wavelength [1]:

$$
\frac{\Delta \lambda(T)}{\lambda\left(T_{0}\right)} \approx \sum_{i} \frac{\Delta n_{i}(T)}{n_{i}\left(T_{0}\right)} \times f r(i)
$$

Here the summation index denotes silicon or elastomer, $n_{i}\left(T_{0}\right)$ and $\Delta n_{i}(T)$ are the refractive index at the reference temperature $\left(20^{\circ} \mathrm{C}\right)$ and the index perturbation resulting from heating the material to temperature $T$. Further, $f r(i)$ is the fraction of the electrical energy of the cavity mode in the region where the perturbation occurs. Simply putting $f r(S i)+f r($ elast $)=1$, and using Eq. 1 combined with $n_{\text {elast }}(T)$ and $n_{S i}(T)$ we obtain $f r(S i)=0.66$ and $f r($ elast $)=0.34$. So, thermal tuning effect we observe results from the combination of $34 \%$ of the total energy of the cavity mode with the negative TOC of the elastomer.

The aforementioned discrepancies for the position of the band edge and the resonance, and for the resonance shift with temperature, suggests that the experiment probes effective values of the index and the TOC of the elastomer that are systematically some lower than the regular values. By performing further simulations we find that for the band edge and resonance position the discrepancy is to a large extent removed by using $n_{\text {eff }}=0.92 \times n_{\text {elast }}$ (green stripes in Fig. 1(b); stripe positions fitted to experimental positions, with emphasis on the resonance position). Since the cavity mode is more sensitive to the infilled holes than to the claddings, we interpret this reduced index as an indication of partially filled cavity holes. Contrary to holes in non-membrane PhCs, however, where partial filling may arise from air inclusions in the lower part of the holes [16] we suggest that here partial infilling has a different origin. Evaporation of the solvent out of the solution goes along with a density increase, leading to the regular density of the elastomer. In Ref [17], which treats solvent evaporation from large area polymer films, the density increase results from film-thickness reduction. In our case the elastomer solution in the holes is a three-dimensional system with an aspect ratio close to unity, so that size reduction in the lateral direction may be expected as well, a process in which the cohesion forces of the compacting elastomer exceed the adhesion forces to the wall of a hole. Modeling the partial filling of cavity holes with an empty shell $(n=1)$ surrounding a cylindrical elastomeric core, we find $f_{\text {shell }}=0.28$ and $f_{\text {core }}=0.72$ for the filling fractions. In view of the roughness of the model, these fractions show fair agreement with the ratio of the slopes of the lines in Fig. 2(b) for the infilled case, which amount to 0.61 and should be compared to $f_{\text {core }}=0.72$.

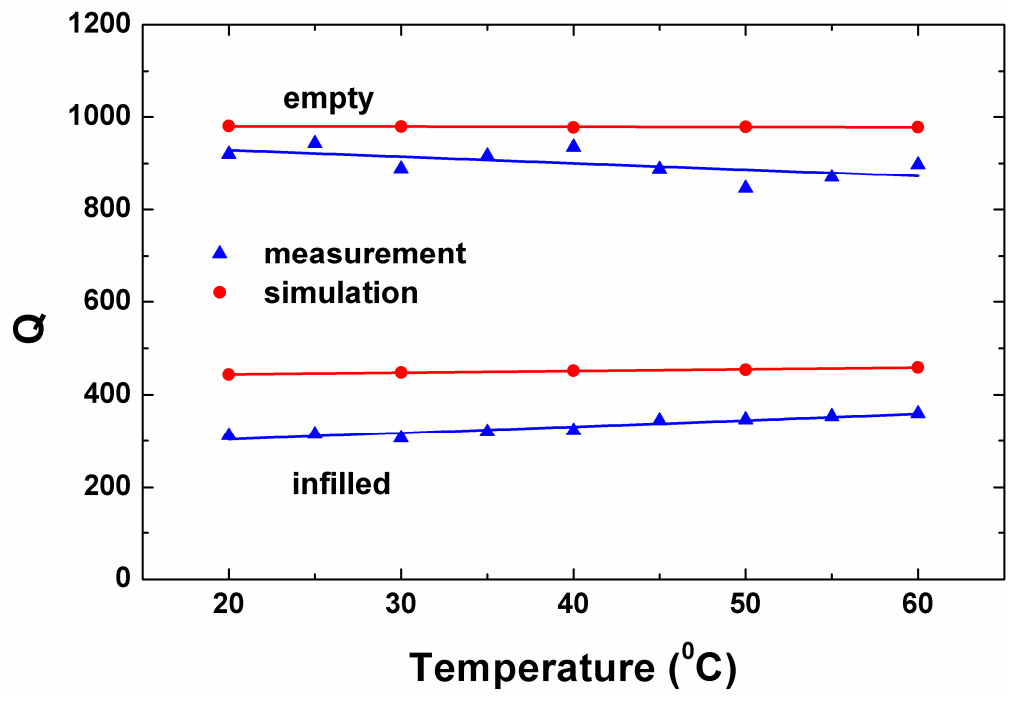

Figure 6. $T$-dependence of the measured and simulated quality factor of the resonance, for the empty and infilled $\mathrm{PhC}$. 
The quality factor $\mathrm{Q}$ is determined from the resonances in Fig. 5(a) for each measurement, using fits to the measured line shape. Figure 6 shows the resulting temperature dependence of Q, for the empty and infilled PhC, along with values obtained from simulations, for which in this case we use MEEP [18] in view of the Q values directly output by the Harminv tool. It is seen that as a result of infilling Q is drastically reduced form about 900 to about 330, while both for the empty case and after infilling Q is rather temperature insensitive. After infilling of the air holes with elastomer, due to the refractive index change from air to elastomer, the $\mathrm{PhC}$ mirror surrounding the cavity have a shallower bandgap and thus lower reflectivity [16]. For infilled cavity, when the temperature increases the cavity shows slight but steady increase of the Q, which arises from the index decrease of the elastomer that leads to a slight increase in the mirror reflectivity. The simulated $\mathrm{Q}$ values are systematically higher than the measured $\mathrm{Q}$ values, which we attribute to fabrication imperfections.

\section{CONCLUSIONS}

In conclusion, global infilling of membrane silicon photonic crystals with the elastomer Kraton SEBS G 1657 and measurement of the transmission of a cavity in an infilled crystal as a function of temperature have been performed. The strong thermal expansion of the elastomer and the resulting negative thermo-optic coefficient of the elastomer lead to a pronounced blue shift on the cavity resonance with temperature. The experimental results are supported by simulations including a somewhat reduced density of the elastomer originating from the constrained geometry of the membrane with holes. These results call for local thermal tuning, for example using dye or quantum dot doping of the elastomer, which enables conversion of locally applied optical energy into heat.

\section{ACKNOWLEDMENT}

The authors gratefully acknowledge the support of the Smart Mix Program of the Netherlands Ministry of Economic Affairs and the Netherlands Ministry of Education, Culture and Science. The authors further thank H.W.M. Salemink, H.M. Ngyen and M.A. Dundar for stimulating discussion, and J. Jackson (Metricon) and A. Houtepen for refractometry and absorbance measurements on bulk elastomer, respectively.

\section{REFERENCES}

[1] Joannopoulos, J.D., S.G. Jonhson, J.N. Winn, and R.D. Meade, [Photonic Crystals Molding the Flow of Light],Princeton University Press, (2008).

[2] Tanabe, T., M. Notomi, S. Mitsugi, A. Shinya, and E. Kuramochi, "All-optical switches on a silicon chip realized using photonic crystal nanocavities", Appl. Phys. Lett. 87(15), 151112, (2005).

[3] Takano, H., Y. Akahane, T. Asano, and S. Noda, "In-plane-type channel drop filter in a two-dimensional photonic crystal slab", Appl. Phys. Lett. 84(13), 2226-2228, (2004).

[4] Painter, O., R.K. Lee, A. Scherer, A. Yariv, J.D. O'Brien, P.D. Dapkus, and I. Kim, "Two-dimensional photonic band-gap defect mode laser", Science. 284(5421), 1819-1821, (1999).

[5] Fushman, I., E. Waks, D. Englund, N. Stoltz, P. Petroff, and J. Vuckovic, "Ultrafast nonlinear optical tuning of photonic crystal cavities", Appl. Phys. Lett. 90(9), -, (2007).

[6] Hopman, W.C.L., A.J.F. Hollink, R.M. de Ridder, K.O. van der Werf, V. Subramaniam, and W. Bogaerts, "Nano-mechanical tuning and imaging of a photonic crystal micro-cavity resonance", Opt. Express. 14(19), 8745-8752, (2006).

[7] Englund, D., B. Ellis, E. Edwards, T. Sarmiento, J.S. Harris, D.A.B. Miller, and J. Vuckovic, "Electrically controlled modulation in a photonic crystal nanocavity", Opt. Express. 17(18), 15409-15419, (2009).

[8] Leonard, S.W., J.P. Mondia, H.M. van Driel, O. Toader, S. John, K. Busch, A. Birner, U. Gosele, and V. Lehmann, "Tunable two-dimensional photonic crystals using liquid-crystal infiltration", Phys. Rev. B. 61(4), R2389-R2392, (2000). 
[9] Polymers, K., http://www.kraton.com/.

[10] Ye, W.N., J. Michel, and L.C. Kimerling, "Athermal high-index-contrast waveguide design", IEEE Photonic Tech. L. 20(9-12), 885-887, (2008).

[11] Leest, M.M.v. and J. Caro, unpublished.

[12] Loncar, M., M. Hochberg, A. Scherer, and Y.M. Qiu, "High quality factors and room-temperature lasing in a modified single-defect photonic crystal cavity", Opt. Lett. 29(7), 721-723, (2004).

[13] Dunsby, C., P.M.P. Lanigan, J. McGinty, D.S. Elson, J. Requejo-Isidro, I. Munro, N. Galletly, F. McCann, B. Treanor, B. Onfelt, D.M. Davis, M.A.A. Neil, and P.M.W. French, "An electronically tunable ultrafast laser source applied to fluorescence imaging and fluorescence lifetime imaging microscopy", J. Phys. D Appl. Phys. 37(23), 3296-3303, (2004).

[14] CrystalWave, http://www.photond.com/products/crystalwave.htm.

[15] Cocorullo, G., F.G. Della Corte, and I. Rendina, "Temperature dependence of the thermo-optic coefficient in crystalline silicon between room temperature and $550 \mathrm{~K}$ at the wavelength of $1523 \mathrm{~nm}$ ", Appl. Phys. Lett. 74(22), 3338-3340, (1999).

[16] Martz, J., R. Ferrini, F. Nuesch, L. Zuppiroli, B. Wild, L.A. Dunbar, R. Houdre, M. Mulot, and S. Anand, "Liquid crystal infiltration of InP-based planar photonic crystals", J. Appl. Phys. 99(10), (2006).

[17] Tsige, M. and G.S. Grest, "Solvent evaporation and interdiffusion in polymer films", Journal of PhysicsCondensed Matter. 17(49), S4119-S4132, (2005).

[18] Oskooi, A.F., D. Roundy, M. Ibanescu, P. Bermel, J.D. Joannopoulos, and S.G. Johnson, "MEEP: A flexible free-software package for electromagnetic simulations by the FDTD method", Comput. Phys. Commun. 181(3), 687-702, (2010). 